\title{
Interrogating the Functional Correlates of Collateralization in Patients with Intracranial Stenosis Using Multimodal Hemodynamic Imaging
}

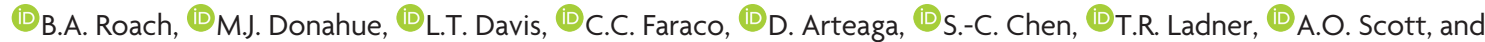

(1) M.K. Strother

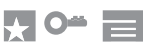

\begin{abstract}
BACKGROUND AND PURPOSE: The importance of collateralization for maintaining adequate cerebral perfusion is increasingly recognized. However, measuring collateral flow noninvasively has proved elusive. The aim of this study was to assess correlations among baseline perfusion and arterial transit time artifacts, cerebrovascular reactivity, and the presence of collateral vessels on digital subtraction angiography.
\end{abstract}

\begin{abstract}
MATERIALS AND METHODS: The relationship between the presence of collateral vessels on arterial spin-labeling MR imaging and DSA was compared with blood oxygen level-dependent MR imaging measures of hypercapnic cerebrovascular reactivity in patients with symptomatic intracranial stenosis $(n=18)$. DSA maps were reviewed by a neuroradiologist and assigned the following scores: 1 , collaterals to the periphery of the ischemic site; 2, complete irrigation of the ischemic bed via collateral flow; and 3, normal antegrade flow. Arterial spin-labeling maps were scored according to the following: 0, low signal; 1, moderate signal with arterial transit artifacts; 2, high signal with arterial transit artifacts; and 3, normal signal.
\end{abstract}

RESULTS: In regions with normal-to-high signal on arterial spin-labeling, collateral vessel presence on DSA strongly correlated with declines in cerebrovascular reactivity (as measured on blood oxygen level-dependent MR imaging, $P<.001$ ), most notably in patients with nonatherosclerotic disease. There was a trend toward increasing cerebrovascular reactivity with increases in the degree of collateralization on DSA $(P=.082)$.

CONCLUSIONS: Collateral vessels may have fundamentally different vasoreactivity properties from healthy vessels, a finding that is observed most prominently in nonatherosclerotic disease and, to a lesser extent, in atherosclerotic disease.

ABBREVIATIONS: ASL = arterial spin-labeling; ATA = arterial transit artifacts; $B O L D=$ blood oxygen level-dependent; $C V R=$ cerebrovascular reactivity; $I C=$ intracranial

$\mathbf{T}$ he presence of intracranial collateral blood flow in steno-occlusive disease has consistently been shown to be one of the most important variables for predicting response to treatment

Received August 3, 2015; accepted after revision November 28

From the Departments of Radiology and Radiological Sciences (B.A.R., M.J.D., L.T.D., C.C.F., D.A., T.R.L., A.O.S., M.K.S.), Neurology (M.J.D.), and Psychiatry (M.J.D.) and the Vanderbilt Center for Quantitative Sciences (S.-C.C.), Vanderbilt Medical Center, Nashville, Tennessee.

This work was supported by National Institutes of Health/National Institute of Neurological Disorders and Stroke grant 5R01NS078828-03 and the American Heart Association grant 14GRNT20150004.

Paper previously presented at: Annual Meeting of the American Society of Neuroradiology and the Foundation of the ASNR Symposium, April 25-30, 2015; Chicago, Illinois.

Please address correspondence to Manus J. Donahue, PhD, Vanderbilt University Institute of Imaging Science, Medical Center North, AAA-3115, 116121 st Ave South, Nashville, TN 37232; e-mail: mj.donahue@vanderbilt.edu

-- Indicates open access to non-subscribers at www.ajnr.org

三 Indicates article with supplemental on-line table

http://dx.doi.org/10.3174/ajnr.A4758 and clinical outcome. ${ }^{1-5}$ Speculations regarding the anatomic and physiologic properties of collateral vessels are frequently debated, and there is still much controversy on how collateralization contributes to prognosis in acute stroke and chronic hypoperfusion. ${ }^{6,7}$ New imaging approaches are being developed with sensitivity to collateralization, yet there is currently insufficient information on how these new techniques relate to objective measures of intracranial circulation in vascular insufficiency. ${ }^{8,9}$

More specifically, intracranial arterioles have the ability to dilate and/or remodel to meet the oxygen-delivery requirements of downstream tissue in response to a vascular insult. The ability of arterioles to modulate cerebral blood volume (milliliters of blood/ milliliters of parenchyma) and cerebral blood flow (milliliters of blood/100 g tissue/min) is commonly referred to as cerebrovascular reactivity (CVR) and can be assessed using functional MR imaging or SPECT techniques during the administration of vasoactive stimuli (eg, hypercapnia or acetazolamide). If the ability of the intracranial circulation to increase $\mathrm{CBV}$ in response to re- 
duced perfusion pressure is inadequate, the oxygen extraction fraction (the ratio of oxygen consumed to oxygen delivered) may increase, and in advanced stages, oxygen metabolism (cerebral metabolic rate of $\mathrm{O}_{2}$, micromole/100 g/min) will decline and an infarct may result.

Collaterals are thought to arise when growth factors, released to reduce metabolic strain, stimulate neoangiogenesis. Collaterals may be recruited from both the affected circulation and uninvolved territories. As the vascular insult evolves, collateral vessels may become more robust, increasing in number and size. ${ }^{10}$ Previous studies have demonstrated that CVR in the presence of steno-occlusive disease and collateralization ranges from reduced to essentially normal compared with healthy tissue. ${ }^{11-14}$ The variability of these findings may relate to the chronicity of the vascular insult and a spectrum of evolving intracranial angiogenesis or remodeling.

The current criterion standard for assessing collateral vessels is digital subtraction angiography, a technique that provides both spatial and temporal information. However, DSA is limited by the inherent procedural risks related to its invasive nature. As our understanding of the importance of collaterals evolves, a less invasive and more time-efficient measure of collaterals is needed to guide treatment decisions. Multiphase CTA, which assesses intracranial collaterals via additional delayed equilibrium and late venous phase acquisitions, has recently been shown to be useful in triaging patients with stroke compared with single-phase CTA. ${ }^{15}$ As with DSA, however, multiphase CTA is constrained by radiation dose concerns and limitations related to intravenous contrast requirements.

Alternatively, information regarding collateralization can be derived from noninvasive $\mathrm{CBF}$-weighted arterial spin-labeling (ASL) or blood oxygenation-weighted blood oxygen level-dependent (BOLD) MR imaging. In ASL, blood water protons are labeled magnetically; this feature creates an endogenous tracer that can be used to assess CBF and blood arrival times on the timescale of the longitudinal blood water relaxation time $(\mathrm{T} 1 \sim$ 1.65 seconds at $3 \mathrm{~T}) .{ }^{16}$ At typical so-called postlabeling delay times of 1.5-2 seconds, labeled blood water exchanges with tissue and creates a small $(1 \%-2 \%)$ perfusion signal. However, when the arterial arrival time is much longer, as is the case in collateral vessels, the label will remain in the vasculature and create wellknown arterial transit artifacts (ATA, signal $>2 \%$ ). Thus, temporal vascular information can be provided in the presence of parenchyma with collateral vessels. Recently, it has been shown that ASL MR imaging can provide information similar to that of DSA for assessing the presence and intensity of collateral flow. ${ }^{17,18}$

Additionally, more common BOLD MR imaging can provide complementary qualitative information, often with higher temporal resolution and reduced insensitivity to slow blood arrival than ASL. In evoked BOLD MR imaging, T2* images are obtained during periods of a vasodilatory stimulus such as hypercapnia. Here, the larger increases in CBF and arterial CBV in relation to small-to-negligible changes in the cerebral metabolic rate of $\mathrm{O}_{2}$ lead to a higher fractional concentration of oxyhemoglobin relative to deoxyhemoglobin in capillaries and veins, which translates to a lengthening of the surrounding blood and tissue water $\mathrm{T} 2^{*}$ and an increase in the MR signal. BOLD MR imaging can be performed at much higher temporal resolution than ASL (eg, 1-2 seconds for BOLD relative to $4-8$ seconds for ASL), yet its contrast is more qualitative, with contributions primarily from $\mathrm{CBF}$, $\mathrm{CBV}$, and the cerebral metabolic rate of $\mathrm{O}_{2}$.

The primary aim of this study was to assess the presence and intensity of collateral vessels by using baseline ASL MR imaging and DSA hemodynamic imaging in sequence with hypercapnic BOLD MR imaging in the setting of steno-occlusive disease and, specifically, to understand the vascular compliance of collateral vessels. The hypothesis investigated was that parenchyma perfused by collateral vessels has reduced CVR relative to healthy parenchyma.

\section{MATERIALS AND METHODS Participants}

Patients ( $n=18$; mean age, 49 years; age range, $25-71$ years; 10 women, 8 men) were recruited as part of a prospective longitudinal clinical trial (Vanderbilt Assessment of Multimodal MRI in Patients at-Risk for stroke with Intracranial Stenosis) that has been approved by the institutional review board. Patients provided informed, written consent as required by the local institutional review board on presentation with symptoms consistent with ischemic cerebrovascular disease. Those selected for this study either presented with atherosclerotic intracranial (IC) stenosis ( $n=9$; mean age, 56 years; 3 women and 6 men) or nonatherosclerotic IC stenosis $(n=9 ; 7$ with Moyamoya disease, 1 with fibromuscular dysplasia, and 1 with Takayasu arteritis; mean age, 42 years; 7 women and 2 men). Hypercapnic BOLD and baseline ASL MR imaging was performed within 30 days of the DSA acquisition. Imaging in all patients was performed before revascularization.

\section{MR Imaging}

Patients were scanned at 3T (Achieva; Philips Healthcare, Best, the Netherlands) by using body coil transmission and neurovascular 16-channel sensitivity encoding reception.

Patients underwent a multimodal imaging protocol consisting of the following scans: 1 ) T1-weighted (MPRAGE: $1 \times 1 \times 1$ $\mathrm{mm}^{3} ; \mathrm{TR} / \mathrm{TE}=8.9 / 4.6 \mathrm{~ms}$; duration $=3$ minutes 47 seconds $) ; 2$ ) T2-weighted FLAIR $\left(0.9 \times 0.9 \times 1 \mathrm{~mm}^{3}\right.$; TR/TE $=11,000 / 120$ $\mathrm{ms}$; multishot turbo spin-echo inversion recovery; duration $=1$ minute 39 seconds); 3) baseline (normocapnic normoxic) CBFweighted pseudocontinuous ASL $\left(3.5 \times 3.5 \times 7 \mathrm{~mm}^{3}\right.$; TR/TE/ postlabeling delay times $=4500 / 11 / 1600 \mathrm{~ms} ; 17$ sections; ascending acquisition; 1500-ms Hanning-windowed pulse train; 90-mm labeling offset); and 4) hypercapnic BOLD (single-shot gradientecho EPI with TR/TE $=2000 / 35 \mathrm{~ms}$, duration $=12$ minutes ). For BOLD, patients were fitted with a nasal cannula to measure endtidal $\mathrm{CO}_{2}$ levels and a nonrebreathing mask to supply medicalgrade room air $\left(21 \% \mathrm{O}_{2}, 79 \% \mathrm{~N}_{2}\right)$ or a carbogen mixture $(5 \%$ $\mathrm{CO}_{2}, 95 \% \mathrm{O}_{2}$ ); other patient vitals (eg, peripheral arterial oxygen saturation, heart rate, and blood pressure) were monitored by a respiratory therapist. A 2-block paradigm consisting of 180-second blocks of breathing carbogen interleaved with a block of breathing room air was used during the BOLD MR imaging acquisition, with 90 -second blocks of room air breathing at the beginning and end of the paradigm. Quantitative differences be- 


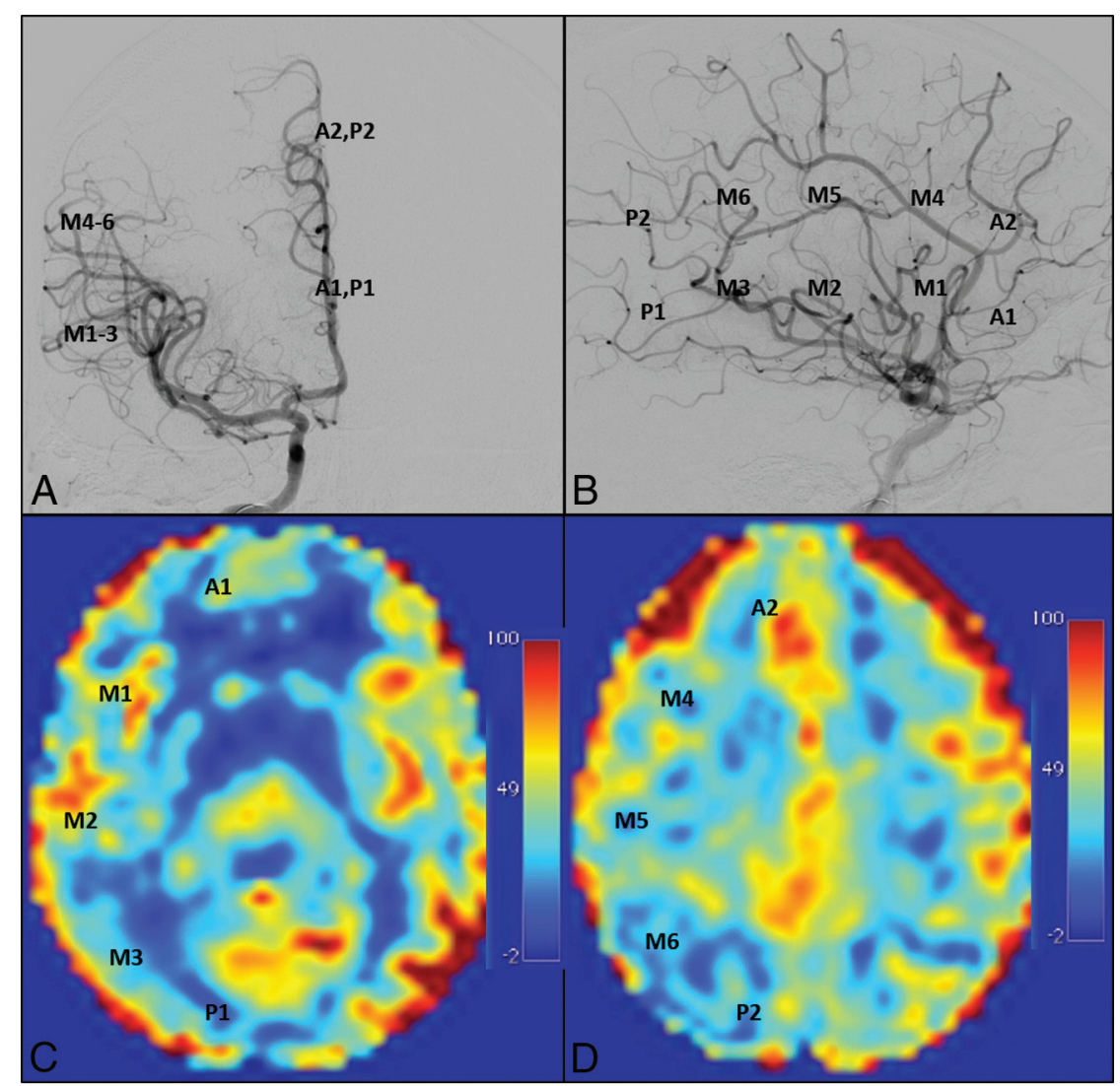

FIG 1. ASPECTS territories superimposed on normal DSA and ASL MR images. $A$ and $B$, Territories superimposed on anteroposterior and lateral projections during intracranial ICA angiography. $C$ and $D$, Territories superimposed at the ganglionic and supraganglionic levels, respectively. control images. CBF maps were quantified in absolute units (milliliters/100 g/min) by applying a 2-compartment perfusion model by using constrained nonlinear optimization with identical parameters as outlined in the literature. ${ }^{21,22} \mathrm{CBF}$ maps were transformed to standard space (4-mm isotropic resolution) by applying the same affine transformation matrix calculated from the BOLD images.

Twenty brain regions in territories defined by the Alberta Stroke Programme Early CT Score criteria ${ }^{18}$ were considered in each subject (eg, 360 regions evaluated across all volunteers).

\section{Collateral Grading}

A neuroradiologist (M.K.S., with 10 years' experience), blinded to ASL findings, graded the presence and intensity of collateral vessels on DSA in 20 territories defined by the ASPECTS criteria (Fig 1). ${ }^{18}$ A previously established 4-point scoring system was used as follows: 0 , no collaterals visible; 1 , collaterals to the periphery of the ischemic site; 2 , complete irrigation of the ischemic bed with collateral flow; and 3, normal antegrade flow. Flow was then assessed in the corresponding anatomic locations tween carbogen and a simpler hypercapnic normoxic stimulus have been reported in the literature. ${ }^{19}$

\section{Digital Subtraction Angiography}

DSA was performed clinically in the neuroangiography suite by using an Allura Xper biplane neuro x-ray system (Philips Healthcare). Selected catheterizations of carotid and vertebral arteries were performed in multiple projections by using nonionic, watersoluble intra-arterial contrast. Stenoses were graded on DSA by using established criteria by a board-certified neuroradiologist (M.K.S.). ${ }^{20}$

\section{BOLD and ASL Analysis}

MR imaging data were analyzed by using an in-house Matlab (MathWorks, Natick, Massachusetts) code and routines available from the fMRI of the Brain Software Library (FSL; http://www. fmrib.ox.ac.uk/fsl). First, affine motion correction, linear slice time correction, and spatial smoothing (full width at half maximum $=6 \mathrm{~mm}$ with a Gaussian kernel) were applied. Baseline drift correction was performed on a voxelwise basis by quadratic polynomial regression. Subsequently, functional data were coregistered to a standard atlas (Montreal Neurological Institute; spatial resolution $=4 \mathrm{~mm}$ isotropic) to enable spatial comparison across subjects. $Z$-statistic and signal change maps were calculated from the BOLD data as has been described in the literature. ${ }^{19}$

All cerebral blood flow quantification was performed in Matlab. Surround subtractions were performed between the label and on ASL MR images by 3 separate graders ( 2 board-certified neuroradiologists, M.K.S. and L.T.D., and 1 imaging physicist, M.J.D.), blinded to non-ASL findings. A similar scoring system was used for ASL MR imaging: 0, no or minimal ASL signal; 1, moderate ASL signal with ATA; 2 , high ASL signal with ATA; and 3 , normal perfusion without ATA. ${ }^{8,17,23}$ Scoring was then converted to a simple 2-point system: 0 , collaterals absent; 1 , collaterals present. A score of 1 (presence of collaterals) was assigned to regions with DSA or ATA scores of 1 or 2, while a score of zero (absence of collaterals) was assigned to regions with DSA or ATA scores of 0 or 3 . A representative image with ATA in the right M3 region correlating with collaterals on DSA is shown in Fig 2.

\section{Statistical Analysis}

The first aim was to understand the similarities between the collateral grading from ASL and DSA. Due to the relative novelty of the ASL scoring system, interreader agreement was first evaluated with Cohen $\kappa$ analysis with the common criteria: poor agreement, $0-0.20$; fair agreement, $0.20-0.40$; moderate agreement, $0.40-$ 0.60 ; good agreement, $0.60-0.80$; very good agreement, $0.80-$ 1.00. The analysis was performed for the binary scoring system. Analyses were also performed separately for atherosclerosis-versus-nonatherosclerosis subtypes.

Next, to understand the CVR in regions with collaterals, territories with or without collaterals were placed into 2 groups by using DSA. CVR values were normalized to the cerebellum in the 


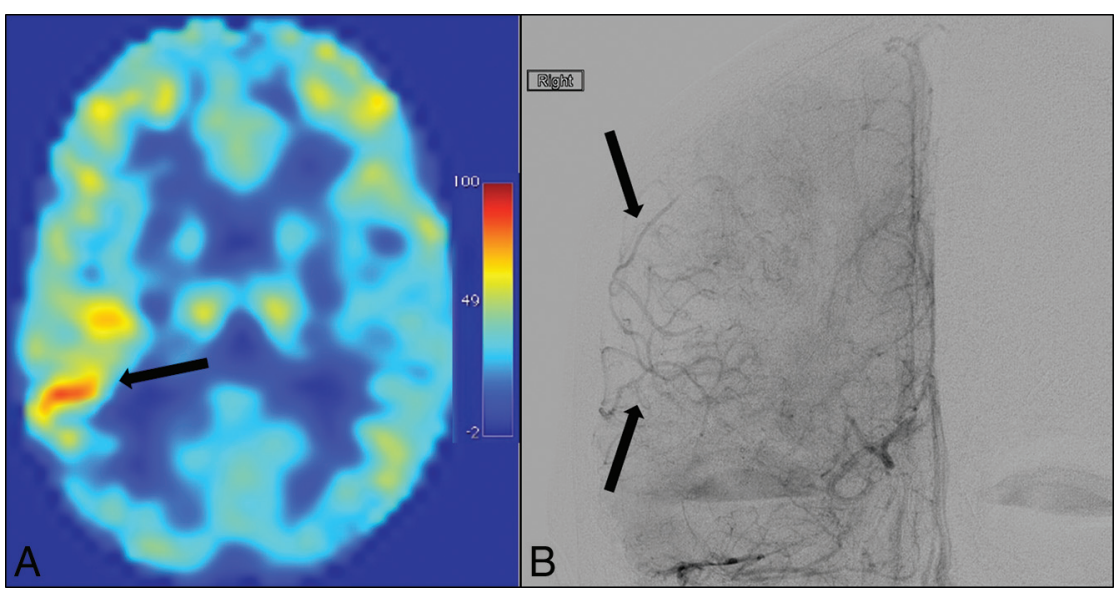

FIG 2. A, Arterial transit artifacts (arrow) at the ganglionic level on ASL MR imaging in the M3 region (ASPECTS designation criteria) representing leptomeningeal collateralization. $B, A$ similar distribution is shown in an early venous phase anteroposterior right ICA angiogram showing leptomeningeal collaterals (arrows) arriving at the periphery of the ischemic site.

Table 1: Modified Suzuki scale

\begin{tabular}{ll}
\hline Score & Description of Classification \\
\hline 0 & No evidence of disease \\
I & Mild-to-moderate stenosis around the ICA bifurcation \\
& with absent or slightly developed ICA MMD \\
II & Severe stenosis around the ICA bifurcation or occlusion \\
& of either proximal anterior or MCA branches with \\
& well-developed ICA MMD \\
III & Occlusion of both anterior and MCA branches with \\
& well-developed ICA MMD \\
IV $\quad$ Complete occlusion of both anterior and MCA branches \\
\end{tabular}

Note:-MMD indicates Moyamoya disease.

Table 2: Collaterals on ASL (presence of ATA) versus DSAconfirmed collaterals-nonatherosclerosis

\begin{tabular}{llcc}
\hline & & \multicolumn{2}{c}{ DSA } \\
\cline { 3 - 4 } & & Yes & No \\
\hline ASL & Yes & 48 & 50 \\
& No & 9 & 25 \\
& $P=.022\left(\chi^{2}\right)$ & \\
& Sensitivity, $84.2 \%(78.0 \%-90.4 \%)$ & \\
& Specificity, 33.3\% (25.3\%-41.4\%) & \\
& PPV, 49.0\% (40.5\%-57.5\%) & \\
& NPV, $73.5 \%(66.0 \%-81.1 \%)$ & \\
\end{tabular}

Note:-PPV indicates positive predictive value; NPV, negative predictive value.

Table 3: Collaterals on ASL (presence of ATA) versus DSAconfirmed collaterals-atherosclerosis

\begin{tabular}{llcr}
\hline & & \multicolumn{2}{c}{ DSA } \\
\cline { 3 - 4 } & & Yes & No \\
\hline ASL & Yes & 24 & 130 \\
& No & 9 & 44 \\
& $P=.811\left(\chi^{2}\right)$ & \\
& Sensitivity, $72.7 \%(65.1 \%-80.3 \%)$ & \\
& Specificity, $25.3 \%(17.9 \%-32.7 \%)$ & \\
& PPV, 15.6\% (9.4\%-21.8\%) & \\
& NPV, 83.0\% (76.6\%-89.4\%) & \\
\hline
\end{tabular}

Note:-PPV indicates positive predictive value; NPV, negative predictive value.

corresponding territories and compared by using an unpaired 2 -tailed $t$ test, separately for all patients, patients with atherosclerosis, and those without atherosclerosis to understand whether
CVR trends are different between subgroups. To compare the influence that collateralization has on measures of hemodynamic reserve, we separated territories into 4 groups based on the DSA score, and corresponding mean normalized cerebrovascular reactivity values were calculated. An unpaired 2-tailed $t$ test with $P<.05$ was applied to assign significance.

\section{RESULTS}

\section{Patient Characteristics}

Patient demographics and severity/extent of disease, including the presence and location of infarction, are detailed in the On-line Table. No areas of infarction occupied $>50 \%$ of any individual territory analyzed; infarct volume was generally much smaller than the territory volume considered. Table 1 illustrates the components of the modified Suzuki Scale score. ${ }^{24,25}$

\section{Collateral Grading}

For the 3 raters, ASL scores between raters were found to have fair agreement $(\kappa=0.31-0.36)$ for patients with and without atherosclerosis when raters 1 and 2 were compared (both board-certified neuroradiologists). Raters 1 and 3 (a board-certified neuroradiologist and an imaging physicist) provided moderate agreement $(\kappa=0.48-$ 0.56). Due to variation in the ASL scoring, the more standardized DSA scoring metric was used for all further comparisons of the effect of collaterals on cerebrovascular reactivity (Tables 2 and 3).

\section{Collateral Effect on Functional Hemodynamic Imaging (BOLD MR Imaging)}

As shown in Fig 3, territories perfused by collateral vessels on DSA that also had ASL scores of $>0$ demonstrated significantly decreased CVR $(P<.001)$. When considered separately, regions in patients with nonatherosclerotic IC stenosis demonstrated significantly decreased CVR compared with normally perfused regions $(P=.022)$, while the atherosclerosis subset showed no significant correlation $(P=.338)$.

\section{Influence of Collateralization on Measures of Hemodynamic Reserve}

The number of regions considered for patients with atherosclerosis was $5($ DSA score $=0), 39($ DSA score $=1), 28($ DSA score $=$ 2 ), and 68 (DSA score $=3$ ) for patients without atherosclerosis and $0($ DSA score $=0), 16($ DSA score $=1), 25($ DSA score $=3)$, and 179 (DSA score $=3$ ) for the patients with atherosclerosis. Due to the low number of subjects with a DSA score of zero, this group was not considered statistically.

When territories were separated on the basis of the DSA score for collateralization (Fig 4), CVR was significantly decreased in regions with both collaterals to the periphery of the ischemic site (DSA $=1, P<.05)$ and complete irrigation of the ischemic bed with collateral flow (DSA $=2, P<.05)$ compared with normally perfused territories (DSA $=3$ ). A positive trend was shown when comparing CVR between territories with scores of DSA $=1$ and 

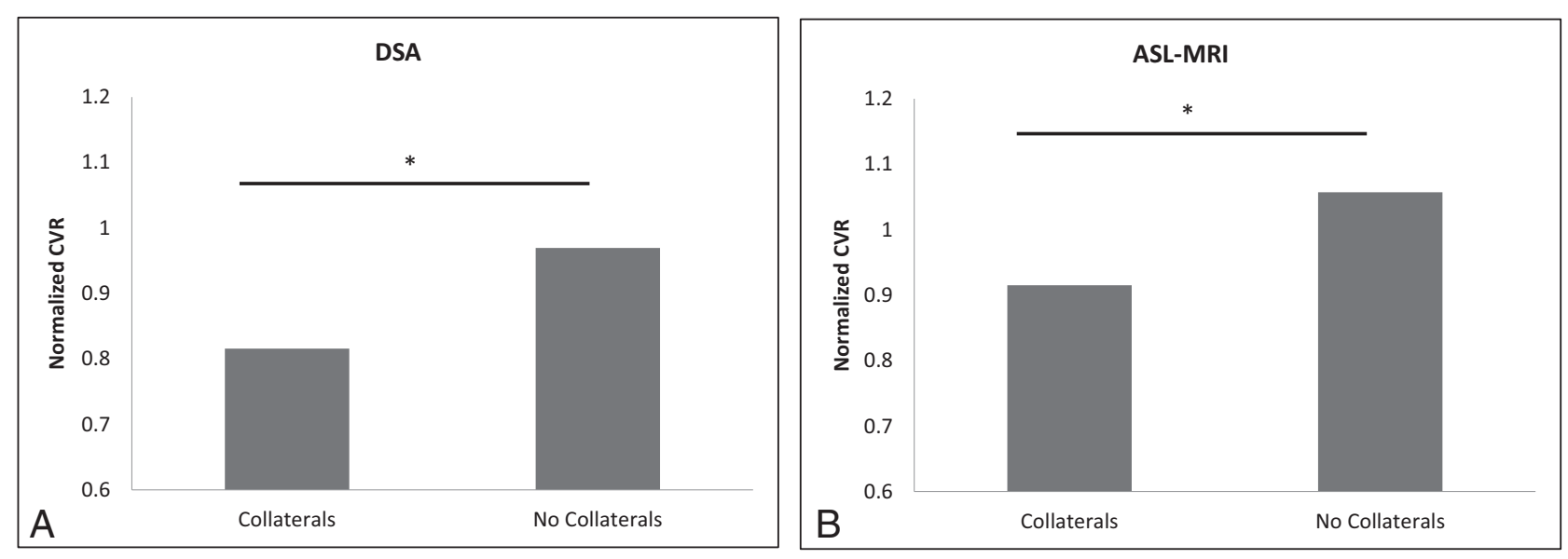

FIG 3. CVR normalized to the cerebellum ( $y$-axis) is shown to be significantly decreased in regions perfused by collaterals on both DSA ( $A$ ) and ASL MR imaging $(B)(P<.001$ and $P=.0012$, respectively).

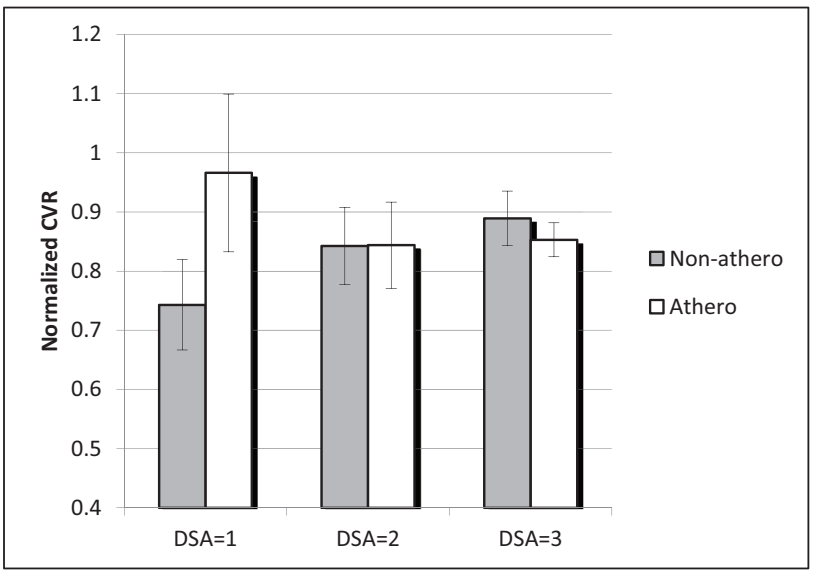

FIG 4. CVR is known to be decreased in the presence of collaterals; however, the graph above shows that CVR tends to increase along with increases in the degree of collateralization. These findings are generally driven by the nonatherosclerotic IC stenosis group.

DSA $=2(2$-sided $P=.082)$. Findings were driven by the nonatherosclerotic group.

Representative DSA and ASL MR images from a patient with Takayasu arteritis, who presented with stroke-like symptoms from TIA as a result of near-occlusive cervical ICA stenosis caused by intimal dissections (Fig 5), demonstrated both primary and secondary collateral pathways, which developed due to flow-limiting cervical ICA stenosis.

\section{DISCUSSION}

The primary findings of this study are the following: 1) ATA from ASL MR imaging correlated significantly with collateral perfusion on DSA in patients with nonatherosclerotic IC stenosis and demonstrated a positive trend in patients with atherosclerotic IC stenosis; 2) regions with collaterals had significantly decreased CVR compared with regions without collaterals; and 3) in regions with collaterals, CVR increased with increasing levels of collateralization.

Several prior studies have examined the ability of ASL MR imaging to assess collaterals compared with DSA, with results supporting our findings. ${ }^{8,17,26}$ However, 1 study expressed difficulty with ASL interpretation and found no correlation with DSA for collaterals. ${ }^{27}$
This study had a small patient subset $(n=7)$ with IC stenosis resulting from both Moyamoya disease $(n=3)$ and atherosclerosis $(n=$ 4). We observed a similar result in a mixed atherosclerotic and nonatherosclerotic population, showing only a trend toward correlation with DSA. However, a subgroup analysis showed that the atherosclerotic group was masking a statistically significant correlation in the nonatherosclerotic group. This finding suggests that ASL MR imaging analysis for collaterals may be more reliable in the nonatherosclerotic population.

Differences between the current study and those previously reported in the literature may be driven by variability in collateral scoring by territory, most notably in the ASL scores. This variability is demonstrated by only a fair-to-moderate interrater agreement with respect to ASL scoring in the current study. A few experimental reasons potentially explain these results, likely from the variability of the arrival-time delays and the sensitivity of the ASL method used between studies. For instance, Zaharchuk et $\mathrm{al}^{8}$ used a 1.5T pseudocontinuous ASL sequence with a long postlabeling delay of 2 seconds. Due to the shorter blood $\mathrm{T} 1$ recovery time at $1.5 \mathrm{~T}(\mathrm{~T} 1 \sim 1.2-1.4$ seconds) relative to $3 \mathrm{~T}$ ( $\mathrm{T} 1 \sim 1.6-1.8$ seconds) used in our study, it is possible that voxels with large endovascular signal were more noticeable relative to those with tissue perfusion because tissue $\mathrm{T} 1$ is much shorter $(<1$ second at $1.5 \mathrm{~T}$, depending on the white and gray matter fraction). ${ }^{8}$ Chng et al ${ }^{17}$ used a pulsed ASL approach at 3T with multiple delay times, which is known to be more sensitive to variation in blood-arrival times. Therefore, it is likely that ASL methods and postlabeling delay times should be adjusted for specific sensitivity to arrival-time delays. Imaging parameters used in our study were optimized for perfusion, which may not be as sensitive to the specific range of delays observed in our patients. It is likely that different ASL protocols have different discriminatory abilities to detect these effects, which also may depend on the patient population and range of blood water arrival times.

Several findings may underlie the improved correlation of collaterals and CVR from patients without atherosclerosis (largely those with Moyamoya disease) compared with those with atherosclerosis. It has been shown that regions supplied by primary collateral pathways (via communicating arteries through the circle of Willis) tend to have better CVR to hypoperfused territories, even approaching normal in 1 study. ${ }^{14}$ 


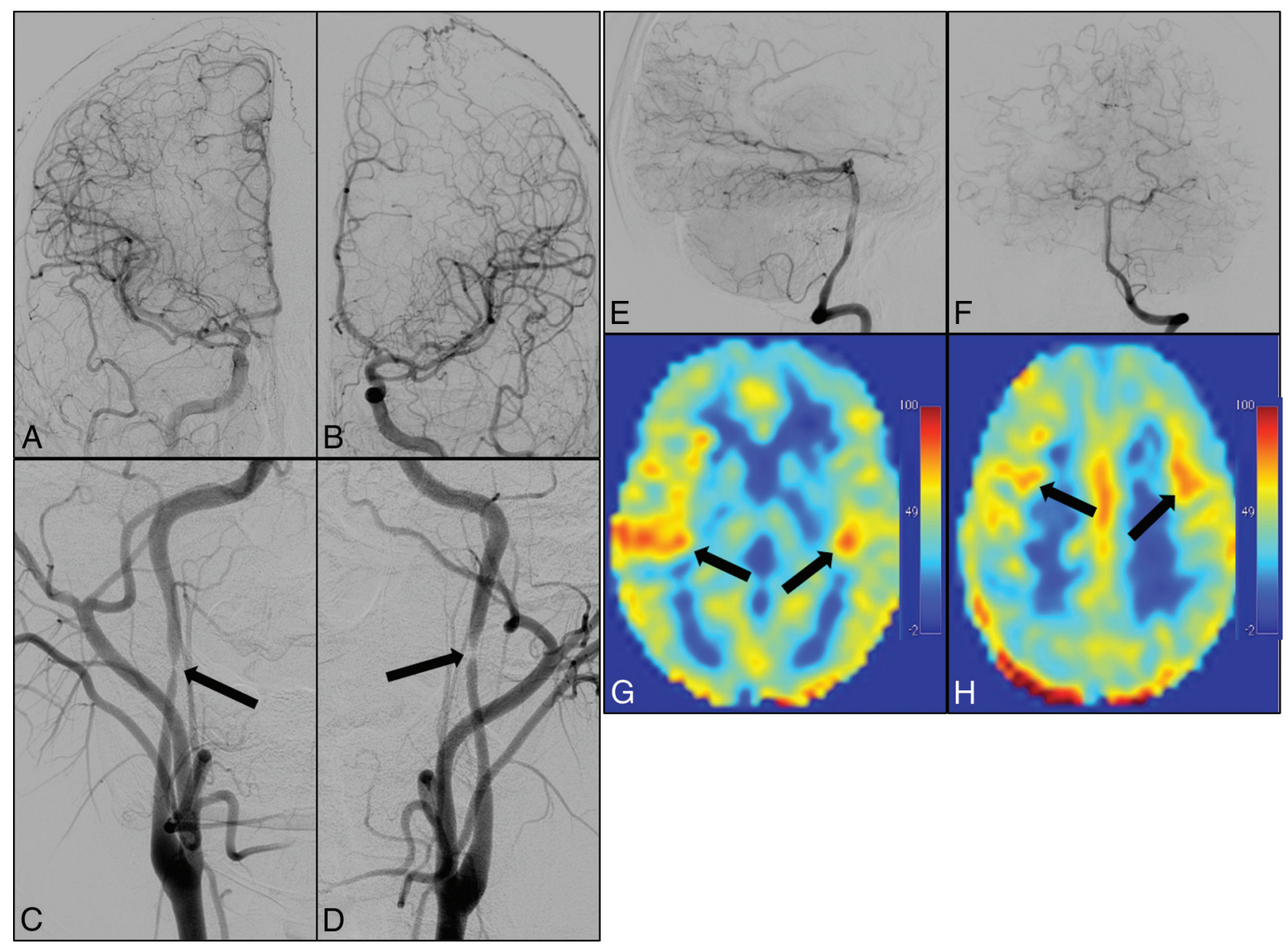

FIG 5. DSA images after injection of the ICA $(A)$, common carotid artery $(B)$, and vertebral artery $(E$ and $F)$ show multiple deep and leptomeningeal secondary collateral pathways. Collateralization is a result of poor intracranial flow due to high-grade stenoses of the cervical ICAs (arrows, $C$ and $D$ ), shown after common carotid injection in the anteroposterior dimension. Correlative signal is shown on ASL MR images at the ganglionic (arrows, $G$ ) and supraganglionic (arrows, $H$ ) levels.

The subjects with atherosclerotic IC stenosis in our study were more likely to have isolated proximal disease (4 of 9 patients with atherosclerosis compared with 1 of 9 without it), which may preserve primary collateral pathways. Collateral flow via intact primary collaterals is not represented by ATA on ASL. It is suggested that secondary collateral pathways only become established when these primary pathways fail to sufficiently augment flow. These primary pathways are often not intact in the nonatherosclerotic group, creating more reliance on the secondary collaterals, which would be closer to autoregulatory capacity, depending on the severity of stenosis. In Moyamoya disease, collaterals have been shown to correlate with disease severity. ${ }^{28}$

The significant decrease in CVR in territories perfused by collaterals, demonstrated in our study by both ASL and DSA, is consistent with findings on prior studies showing that angiogenesis occurs in response to tissue hypoxia from hypoperfusion. ${ }^{10}$ As suggested by the variability in our data, prior studies have shown a range of decreases in CVR in regions supplied by collateral vessels from markedly decreased to normal compared with normally perfused tissue. ${ }^{11-14}$ This suggests that the presence of collaterals alone does not necessarily portend a protective effect. It is essential to correlate collateralization with a measure of hemodynamic reserve, as can be done with
BOLD MR imaging, to assess the CVR in these hypoperfused territories. Variations in contributions have been attributed to the source of collateralization, with primary pathways (anterior and posterior communicating arteries) resulting in a better protective effect compared with secondary pathways (ophthalmic artery and leptomeningeal vessels). ${ }^{13,14,29-31}$

Clinical implications can be drawn from the current study. First, we add to a growing body of literature supporting the use of ASL MR imaging rather than invasive DSA to assess the presence of collateralization, even for patients with IC stenosis from nonatherosclerotic etiologies. It is imperative that this information be compared with measures of hemodynamic reserve to guide treatment decisions because the presence of collaterals does not necessarily indicate better vascular reserve. Second, we have shown that CVR is decreased in territories perfused by collaterals theoretically approaching zero, in which there is tissue without vascularity compared with normally perfused tissue, but CVR increases with increased collateralization within regions perfused by collaterals. Finally, our data suggest more variability within the atherosclerosis subset regarding how collateralization is achieved and the contribution of collaterals toward CVR. This suggestion is largely speculative, however, because our sample size limited definitive comparison of circle of Willis integrity within the atherosclerotic subset. 
A limitation of our study is the relatively small patient population used for assessment. This limitation could have some bearing on our data variability, particularly in the atherosclerosis subset. Additionally, our study and those performed to date have used a standard ASL protocol, which was applied to the entire patient population. However, patients may require an ASL MR imaging protocol with signal acquisition at many different time points to fully evaluate the extent of collateralization, as previously suggested. ${ }^{26}$

\section{CONCLUSIONS}

Our study demonstrates that ASL MR imaging may provide a useful noninvasive measure of collateralization, particularly in the nonatherosclerotic IC stenosis subset. Furthermore, collaterals identified on ASL MR imaging with ATA correlated with decreased CVR compared with regions not perfused via collaterals. Finally, within territories perfused by collaterals, CVR increased with higher DSA scores.

Disclosures: Brent A. Roach—RELATED: Grant: National Institutes of Health/National Institute of Neurological Disorders and Stroke grant 5R01NS078828-03,* American Heart Association grant 14GRNT20150004. * Manus J. Donahue-RELATED: Grant: National Institutes of Health/National Institute of Neurological Disorders and Stroke, ${ }^{*}$ Comments: National Institutes of Health/National Institute of Neurological Disorders and Stroke R01 to support experimental costs and coinvestigator salaries. Daniel Arteaga_RELATED: Grant: American Heart Association grant 14PRE20370055, Radiological Society of North America grant RMS1402; Support for Travel to Meetings for the Study or Other Purposes: American Heart Association grant 14PRE20370055; Fees for Participation in Review Activities such as Data Monitoring Boards, Statistical Analysis, Endpoint Committees, and the Like: part of a grant used to fund statistical analysis, National Institutes of Health/National Institute of Neurological Disorders and Stroke grant IR01NS078828. * Megan K. Strother-RELATED: Grant: National Institutes of Health.* *Money paid to the institution.

\section{REFERENCES}

1. Bang OY, Saver JL, Kim SJ, et al. Collateral flow predicts response to endovascular therapy for acute ischemic stroke. Stroke 2011;42: 693-99 CrossRef Medline

2. Sheth SA, Liebeskind DS. Imaging evaluation of collaterals in the brain: physiology and clinical translation. Curr Radiol Rep 2014; 2:29 CrossRef Medline

3. Lima FO, Furie KL, Silva GS, et al. The pattern of leptomeningeal collaterals on CT angiography is a strong predictor of long-term functional outcome in stroke patients with large vessel intracranial occlusion. Stroke 2010;41:2316-22 CrossRef Medline

4. Bang OY, Saver JL, Buck BH, et al. Impact of collateral flow on tissue fate in acute ischaemic stroke. J Neurol Neurosurg Psychiatry 2008; 79:625-29 Medline

5. Liebeskind DS. Collaterals in acute stroke: beyond the clot. Neuroimaging Clin North Am 2005;15:553-73 CrossRef Medline

6. Brozici M, van der Zwan A, Hillen B. Anatomy and functionality of leptomeningeal anastomoses: a review. Stroke 2003;34:2750-62 CrossRef Medline

7. Seeta Ramaiah S, Churilov L, et al. The impact of arterial collateralization on outcome after intra-arterial therapy for acute ischemic stroke. AJNR Am J Neuroradiol 2014;35:667-72 CrossRef Medline

8. Zaharchuk G, Do HM, Marks MP, et al. Arterial spin-labeling MRI can identify the presence and intensity of collateral perfusion in patients with moyamoya disease. Stroke 2011;42:2485-91 CrossRef Medline

9. Donahue MJ, Strother MK, Hendrikse J. Novel MRI approaches for assessing cerebral hemodynamics in ischemic cerebrovascular disease. Stroke 2012;43:903-15 CrossRef Medline

10. Lehoux S, Lévy BI. Collateral artery growth: making the most of what you have. Circ Res 2006;99:567-69 CrossRef Medline

11. Bokkers RP, van Osch MJ, Klijn CJ, et al. Cerebrovascular reactivity within perfusion territories in patients with an internal carotid artery occlusion. J Neurol Neurosurg Psychiatry 2011;82:1011-16 CrossRef Medline
12. Norrving B, Nilsson B, Risberg J. rCBF in patients with carotid occlusion. Resting and hypercapnic flow related to collateral pattern. Stroke 1982;13:155-62 CrossRef Medline

13. Müller M, Schimrigk K. Vasomotor reactivity and pattern of collateral blood flow in severe occlusive carotid artery disease. Stroke 1996;27:296-99 CrossRef Medline

14. Vernieri F, Pasqualetti P, Matteis M, et al. Effect of collateral blood flow and cerebral vasomotor reactivity on the outcome of carotid artery occlusion. Stroke 2001;32:1552-58 CrossRef Medline

15. Menon B, d'Esterre C, Qazi E, et al. Multiphase CT angiography: a new tool for the imaging triage of patients with acute ischemic stroke. Radiology 2015;275:510-20 CrossRef Medline

16. Lu H, Clingman C, Golay X, et al. Determining the longitudinal relaxation time (T1) of blood at 3.0 Tesla. Magn Reson Med 2004;52: 679-82 CrossRef Medline

17. Chng SM, Petersen ET, Zimine I, et al. Territorial arterial spin labeling in the assessment of collateral circulation: comparison with digital subtraction angiography. Stroke 2008;39:3248-54 CrossRef Medline

18. Barber PA, Demchuk AM, Zhang J, et al. Validity and reliability of a quantitative computed tomography score in predicting outcome of hyperacute stroke before thrombolytic therapy: ASPECTS Study Group-Alberta Stroke Programme Early CT Score. Lancet 2000; 355:1670-74 CrossRef Medline

19. Donahue MJ, Dethrage LM, Faraco CC, et al. Routine clinical evaluation of cerebrovascular reserve capacity using carbogen in patients with intracranial stenosis. Stroke 2014;45:2335-41 CrossRef Medline

20. Samuels OB, Joseph GJ, Lynn MJ, et al. A standardized method for measuring intracranial arterial stenosis. AJNR Am J Neuroradiol 2000;21:643-46 Medline

21. Faraco CC, Strother MK, Dethrage LM, et al. Dual vessel-encoded ASL for simultaneous BOLD and CBF reactivity assessment in patients with ischemic cerebrovascular disease. Magn Reson Med 2015; 73:1579-92 CrossRef Medline

22. Donahue MJ, Faraco CC, Strother MK, et al. Bolus arrival time and cerebral blood flow responses to hypercarbia. J Cereb Blood Flow Metab 2014;34:1243-52 CrossRef Medline

23. Kim JJ, Fischbein NJ, Lu Y, et al. Regional angiographic grading system for collateral flow: correlation with cerebral infarction in patients with middle cerebral artery occlusion. Stroke 2004;35: 1340-44 CrossRef Medline

24. Suzuki J, Takaku A. Cerebrovascular "moyamoya" disease: disease showing abnormal net-like vessels in base of brain. Arch Neurol 1969;20:288-99 CrossRef Medline

25. Mugikura S, Takahashi S, Higano S, et al. Predominant involvement of ipsilateral anterior and posterior circulations in moyamoya disease. Stroke 2002;33:1497-500 CrossRef Medline

26. Wu B, Wang X, Guo J, et al. Collateral circulation imaging: MR perfusion territory arterial spin-labeling at 3T. AJNR Am J Neuroradiol 2008;29:1855-60 CrossRef Medline

27. Chen $\mathrm{H}, \mathrm{Wu} \mathrm{B}, \mathrm{Zhu} \mathrm{G}$, et al. Permeability imaging as a biomarker of leptomeningeal collateral flow in patients with intracranial arterial stenosis. Cell Biochem Biophys 2014 Dec 12. [Epub ahead of print] CrossRef Medline

28. Strother MK, Anderson MD, Singer RJ, et al. Cerebrovascular collaterals correlate with disease severity in adult North American patients with Moyamoya disease. AJNR Am J Neuroradiol 2014;35: 1318-24 CrossRef Medline

29. Ringelstein EB, Weiller C, Weckesser M, et al. Cerebral vasomotor reactivity is significantly reduced in low-flow as compared to thromboembolic infarctions: the key role of circle of Willis. J Neurol Sci 1994;121:103-09 CrossRef Medline

30. Tatemichi TK, Chamorro A, Petty GW, et al. Hemodynamic role of ophthalmic artery collateral in internal carotid artery occlusion. Neurology 1990;40:461-64 CrossRef Medline

31. Rutgers DR, Klijn CJ, Kappelle LJ, et al. A longitudinal study of collateral flow patterns in the circle of Willis and the ophthalmic artery in patients with a symptomatic carotid occlusion. Stroke 2000;31: 1913-20 CrossRef Medline 